\title{
PREDIÇÃO DOS TEORES DE COMPOSTOS FENÓLICOS E FLAVONOIDES NA PARTE AÉREA DAS ESPÉCIES Secale cereale L., Avena strigosa L. E Raphanus sativus L. POR MEIO DE ESPECTROSCOPIA NO INFRAVERMELHO PRÓXIMO (NIR)
}

\author{
Monique Souza $^{\mathrm{a}, *}$, Shirley Kuhnen ${ }^{\mathrm{b}}$, Daniele Cristina da Silva Kazama ${ }^{\mathrm{b}}$, Claudinei Kurtz ${ }^{\mathrm{c}}$, Talita Trapp ${ }^{\mathrm{a}}$, Vilmar Müller \\ Júnior ${ }^{\mathrm{a}}$ e Jucinei José Comin ${ }^{\mathrm{a}}$ \\ aDepartamento de Engenharia Rural, Universidade Federal de Santa Catarina, 88034-000 Florianópolis - SC, Brasil \\ bDepartamento de Zootecnia e Desenvolvimento Rural, Universidade Federal de Santa Catarina, 88034-000 Florianópolis - SC, Brasil \\ 'Estação Experimental da Empresa de Pesquisa Agropecuária e Extensão Rural de Santa Catarina, 88400-000 Ituporanga - SC, Brasil
}

Recebido em 13/04/2017; aceito em 20/07/2017; publicado na web em 12/09/2017

\begin{abstract}
PREDICTION OF CONTENTS OF PHENOLIC COMPOUNDS AND FLAVONOIDS IN AERIAL PART OF Secale cereale L. Avena strigosa L. and Raphanus sativus L. WITH NEAR INFRARED SPECTROSCOPY (NIR). Spectroscopy associated with chemometrics is a non-destructive method of the samples for the prediction of phenolic compounds. The objective of the work was to apply the near infrared spectroscopy (NIR) technique for the prediction of phenolic compounds and flavonoids in aerial part samples of cover crops plants Raphanus sativus L., Secale cereale L. and Avena strigosa L. Samples were collected at 60, 80 and 100 days after sowing (DAS) of the plants and, after lodging (DAA), at 15 and 30 days, in an experiment implanted in the field, in the city of Ituporanga, Santa Catarina. The samples were homogenized, identified, transported on dry ice, lyophilized, crushed, sieved and stored at $-20{ }^{\circ} \mathrm{C}$ for analysis. For the calibration, 84 samples were used and for the validation 10 samples. The combination of NIR spectroscopy and multivariate analysis by partial least squares regression allowed the development of prediction models of phenolics and flavonoids with associated errors averaging 10\%. NIR spectroscopy is recommended for the development of calibration models because it allows to non-destructive infer phenolic and total flavonoid contents, with high analytical frequency, without the use of reagents and other inputs required by the reference method, with results very close to those obtained in the laboratory.
\end{abstract}

Keywords: chemometrics; diffuse reflectance; multivariate analysis; cover crops.

\section{INTRODUÇÃO}

A quantificação de compostos fenólicos e flavonoides totais pode ser realizada via cromatografia ou espectrofotometria via UV-vis pelo método de Folin-Ciocalteau, que se baseia em reações de oxidação e redução entre os compostos e íons metálicos, ${ }^{1}$ porém ambas são análises demoradas, destrutivas e geram resíduos químicos. ${ }^{2,3}$ Métodos que empregam técnicas que proporcionam rápida quantificação de compostos como, por exemplo, o infravermelho próximo (NIR), têm sido utilizados em diversas aplicações em análises químicas. ${ }^{4,5}$

Por meio da espectroscopia no infravermelho próximo, juntamente com métodos quimiométricos, é possível separar os compostos fenólicos, pois obtêm-se informações qualitativas e quantitativas dos constituintes químicos do material. ${ }^{6}$ Além disso, é uma alternativa rápida, precisa, não destrutiva da amostra, de baixo custo e que dispensa o uso de reagentes. ${ }^{4,5} \mathrm{O}$ setor agrícola foi o primeiro a fazer uso intensivo da espectroscopia NIR e, atualmente, esta tem sido aplicada na agricultura de precisão, análise de solos e indústrias químicas. ${ }^{6}$

De acordo com uma pesquisa recente da literatura, até o momento nenhuma pesquisa foi relatada usando NIR para a determinação de compostos fenólicos com o foco em espécies de plantas de cobertura, utilizadas no manejo e adubação de solos. Existem diversos estudos que utilizaram essa técnica para discriminação de plantas, como o arroz, o feijão, o café, o milho e a soja, e nos produtos de origem vegetal, como o azeite de oliva e os sucos de frutas, mas todos voltados para a indústria alimentícia. ${ }^{7-11}$ Trabalhos realizados na área de solos utilizando NIR têm contemplado, por exemplo, nas determinações de nitrogênio total e matéria orgânica em plantas, fósforo e carbono do solo. ${ }^{12-14}$

\footnotetext{
*e-mail: monique_souzaaa@yahoo.com.br
}

Por meio dessa ferramenta, a partir do sinal medido e de combinações de métodos estatísticos com dados químicos obtidos em laboratório, é possível construir modelos de calibração e validação. ${ }^{15}$ A quantificação e a obtenção de espectros, que também pode ser entendida como uma impressão digital do material que está sendo analisado, é utilizada para detectar associações no conjunto de dados, a partir dos quais podem-se estabelecer relações entre as amostras avaliadas e variáveis, além de agrupar ou discriminar as amostras em estudo. ${ }^{15,16}$ Com o desenvolvimento de uma equação de calibração, torna-se possível a construção de uma relação entre os espectros e a propriedade analisada, ou seja, esta equação pode ser usada na predição da composição de amostras desconhecidas, mas pertencentes à população que originou a calibração, com adequado grau de confiabilidade..$^{14,17}$

Além de construir os modelos de calibração para posterior quantificação do conteúdo de compostos fenólicos, é possível inferir se há diferenças entre as amostras e em quais regiões elas diferenciam-se quimicamente. Ao determinarem o conteúdo de compostos fenólicos em plantas de erva mate (Ilex paraguariensis) via NIR, Frizon et al. ${ }^{4}$ mostraram que o NIR, quando combinado com métodos estatísticos, pode ser aplicado como um método eficiente para a quantificação e classificação de amostras em relação à origem de plantio e manejo agronômico adotado nos diferentes produtos.

Nesse contexto, a obtenção de espectros e a construção de curvas de calibração para a predição dos teores de compostos fenólicos com diferentes espécies de plantas de cobertura podem ser alternativas para redução de custos e tempo de análise em laboratório. O uso do NIR, conjuntamente com métodos quimiométricos, permite classificar as amostras em relação à época de plantio e práticas agronômicas, visto que os compostos fenólicos estão ligados à defesa das plantas ${ }^{18}$ e, em sistemas de cultivo, estas informações são relevantes para 
a identificação de qual época a planta produz mais compostos, já que algumas espécies produzem compostos fenólicos com efeito alelopático. ${ }^{19-21}$

O objetivo deste trabalho foi aplicar a técnica da espectroscopia no infravermelho próximo para a predição do conteúdo de compostos fenólicos e flavonoides totais e distinção de amostras de plantas de cobertura Raphanus sativus L., Secale cereale L. e Avena strigosa L. aos 60, 80 e 100 dias após a semeadura a campo e aos 15 e 30 dias após o acamamento das espécies.

\section{PARTE EXPERIMENTAL}

\section{Amostras}

Em 2014 foram realizadas coletas da parte aérea das espécies centeio, nabo-forrageiro e aveia-preta, em experimento implantado a campo na Estação Experimental da Empresa de Pesquisa Agropecuária e Extensão Rural de Santa Catarina (EPAGRI), no município de Ituporanga, região do Alto Vale do Itajaí, Santa Catarina

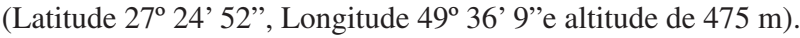

$\mathrm{O}$ centeio e o nabo-forrageiro solteiros e consorciados foram coletados aos 60, 80 e 100 dias após a semeadura das espécies (DAS) e, após o seu acamamento (DAA), aos 15 e 30 dias. A espécie aveia-preta foi coletada somente aos 100 DAS e aos 15 e 30 DAA. Foram coletadas 3 subamostras aleatoriamente de cada parcela para compor uma amostra composta. O delineamento experimental foi em blocos ao acaso com 3 repetições.

As amostras foram identificadas e acondicionadas em tubos do tipo falcon $(50 \mathrm{~mL})$, colocadas em caixa de isopor e transportadas em gelo seco para o laboratório. As amostras foram liofilizadas (modelo L101, Liotop, São Paulo, Brasil) até total remoção da umidade a $-54^{\circ} \mathrm{C}$ e, em seguida, trituradas em moinho, peneiradas $(0,42 \mathrm{~mm}) \mathrm{e}$, posteriormente, novamente acondicionadas em tubos falcons $(50 \mathrm{~mL})$ e conservadas a $-20{ }^{\circ} \mathrm{C}$ para posterior análise.

\section{Solventes}

O metanol (Vetec) $80 \%$ (v/v) foi utilizado como solvente na determinação de fenólicos totais e, para a quantificação, foram preparados os reagentes de Folin-Ciocaltau (Sigma-Aldrich) diluído (1:10) e carbonato de sódio (Vetec) 7,5\% (m/v).

Para a determinação de flavonoides foi utilizado o solvente metanol $80 \%(\mathrm{v} / \mathrm{v})$ e os reagentes cloreto de alumínio (Vetec) $2 \%(\mathrm{~m} / \mathrm{v})$ e álcool etílico (Vetec) para a quantificação. Todas as soluções foram preparadas com água destilada.

\section{Espectroscopia no infravermelho próximo e curvas de calibração}

Para a construção da curva de calibração foram analisadas por espectrômetro infravermelho próximo 84 amostras da parte aérea das espécies nabo-forrageiro, centeio e aveia-preta. Os espectros de reflectância difusa na região do infravermelho foram obtidos em um espectrômetro modelo FT-NIR MPA Bruker (Alemanha), em triplicata na região espectral de 3600 a $12500 \mathrm{~cm}^{-1}$. Foram realizadas 64 varreduras para cada amostra sólida, com resolução de $16 \mathrm{~cm}^{-1}$.

\section{Método de referência}

O método de referência utilizado para a determinação do conteúdo de compostos fenólicos foi o de Folin-Ciocalteau proposto por Singleton e Rossi ${ }^{22}$ e, para flavonoides, a metodologia descrita por Popova et al. ${ }^{23}$
Para extração dos compostos de interesse, $0,1 \mathrm{~g}$ de amostra foi adicionado a solução de metanol $80 \%$ (v/v), seguido de agitação por $2 \mathrm{~h}$, filtração sob vácuo e centrifugação a 4.000 rpm por 10 minutos, coletando-se o sobrenadante. Para determinação do conteúdo de fenólicos totais utilizou-se uma curva padrão externa de ácido gálico (Sigma-Aldrich), expressando os valores em mg de equivalentes de ácido gálico por grama de massa seca $\left(\mathrm{mg} \mathrm{EAG} \mathrm{g}^{-1} \mathrm{MS}\right)$. Do mesmo sobrenadante, foi determinado o conteúdo de flavonoides totais utilizando-se uma curva padrão externa de quercetina (Sigma-Aldrich) e expressando os valores em $\mathrm{mg}$ de equivalentes de quercetina por grama massa seca ( $\mathrm{mg} \mathrm{EQ} \mathrm{g}^{-1} \mathrm{MS}$ ). As análises foram realizadas em triplicatas e as leituras foram realizadas a $425 \mathrm{~nm}$ para flavonoides e $765 \mathrm{~nm}$ pra fenólicos em espectrofotômetro Uv-vis, modelo UV5300PC, Power Supply (China).

\section{Validação do método}

Para a validação do método, em 2015 foram coletadas 10 amostras aleatórias da parte aérea das plantas de cobertura solteiras e consorciadas na mesma área experimental. As amostras foram acondicionadas em tubos do tipo falcon $(50 \mathrm{~mL})$, colocadas em uma caixa de isopor e transportadas em gelo seco para o laboratório, liofilizadas (modelo L101, Liotop, São Paulo, Brasil) até total remoção da umidade a $-54{ }^{\circ} \mathrm{C}$ e, em seguida, trituradas em moinho, peneiradas $(0,42 \mathrm{~mm}) \mathrm{e}$ acondicionadas novamente em tubos falcon $(50 \mathrm{~mL})$ e conservadas a $-20{ }^{\circ} \mathrm{C}$ para posterior análise no infravermelho próximo (NIR). Os espectros foram obtidos em triplicata na região espectral de 3600 a $12500 \mathrm{~cm}^{-1}$, com 64 varreduras para cada amostra sólida e resolução de $16 \mathrm{~cm}^{-1}$.

\section{Análise dos dados e estatística}

Para a importação, pré-tratamento dos dados e construção dos modelos quimiométricos (curvas de calibração e validação) foi utilizado o software Opus Lab Bruker ${ }^{\oplus}$ (v. 7.5). ${ }^{24}$ As calibrações foram realizadas a partir dos espectros originais, e utilizado o modelo de regressão por mínimos quadrados parciais (PLS) para estabelecer uma relação matemática quantitativa entre os dados obtidos pelo método de referência e do NIR.

Para avaliar a precisão da curva, calculou-se o coeficiente de correlação (r) e o erro padrão de calibração (RMSECV) dos dados obtidos pelo de referência (UV-vis) e do NIR.

As amostras classificadas como outliers no gráfico foram detectadas e excluídas dos modelos. Os espectros foram pré-processados empregando os métodos de normalização vetorial (SNV), $1^{\text {a }}$ derivada Savitzky-Golay e segunda derivada.

Os espectros obtidos via NIR também foram submetidos à análise de componentes principais (PCA) e análise de agrupamentos a partir de matrizes de correlação utilizando o software $\mathrm{R}^{\odot} \cdot{ }^{25}$ Os scripts foram escritos em linguagem $\mathrm{R}$ (v. 3.3.1) e disponíveis no pacote specmine ${ }^{26}$ e o relatório de análise de dados gerado automaticamente a partir dos scripts $\mathrm{R}$ utilizando os recursos fornecidos pelo R Markdown.

\section{RESULTADOS E DISCUSSÃO}

\section{Curvas de calibração e análise exploratória}

Inicialmente foi avaliado o conjunto dos espectros originais na região de 3600 a $12500 \mathrm{~cm}^{-1}$ do NIR da parte aérea das espécies Raphanus sativus L., Secale cereale L. e Avena strigosa L., aos 60, 80 e 100 dias após a semeadura a campo e aos 15 e 30 dias após o acamamento (Figuras 1A e 1B). 

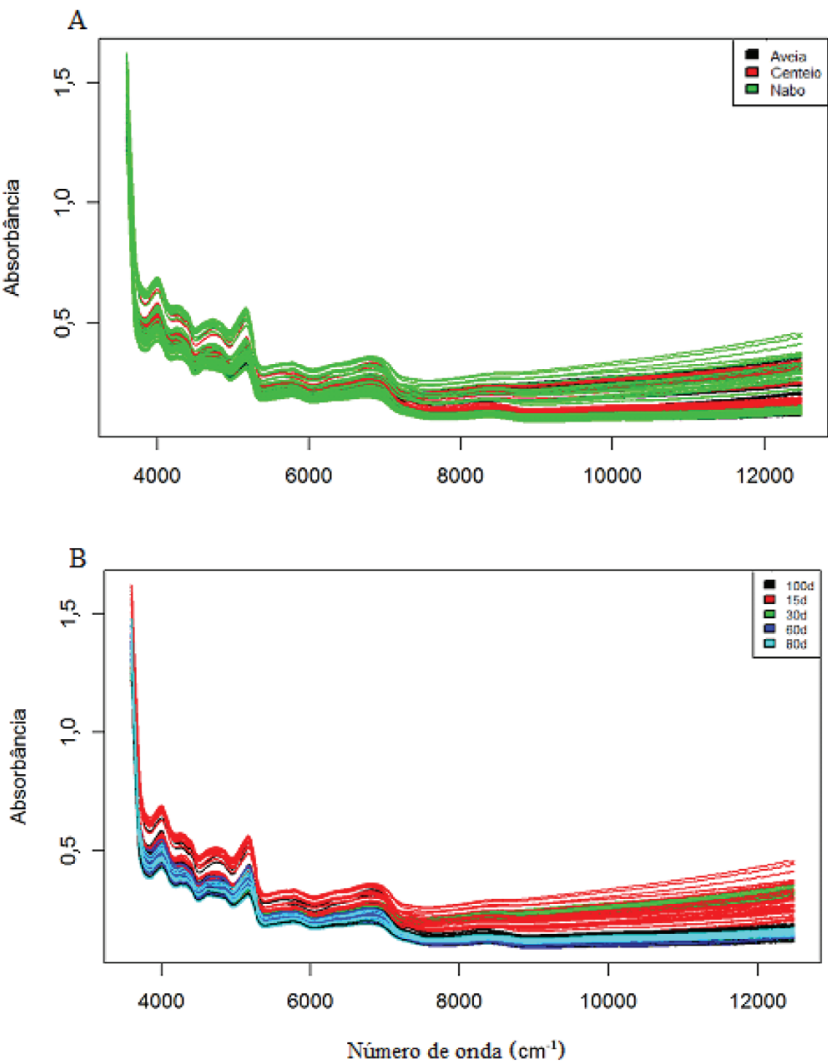

Figura 1. Conjunto dos espectros originais de reflectância difusa na região do NIR, sem transformações matemáticas, da parte aérea das espécies Raphanus sativus L., Secale cereale L. e Avena strigosa L. (A) e espectros nos diferentes tempos de avaliação, aos 60, 80 e 100 dias após a semeadura a campo e aos 15 e 30 dias após o acamamento (B)

A análise dos espectros via NIR revelou perfis similares entre as amostras e maiores valores de absorbância na região em torno de 3600 a $8000 \mathrm{~cm}^{-1}$, isso porque as espécies estudadas apresentaram bandas tipicamente largas, resultado da ocorrência de bandas de combinação e sobretons oriundos das ligações dos grupos C-H, N-H e $\mathrm{O}-\mathrm{H}$, fazendo com que seu espectro seja intenso nesta região do NIR (Figura 1). ${ }^{6,27}$ Também é possível observar na Figura 1 a similaridade entre os espectros das diferentes espécies. Esta sobreposição pode indicar, inicialmente, uma semelhança na composição química das espécies, havendo a necessidade de fazer um pré-processamento dos espectros e o emprego da calibração multivariada para detectar possíveis diferenças. Entretanto, as pequenas discrepâncias entre os espectros indicam, previamente, uma distinção nos perfis da espécie nabo-forrageiro com relação às demais espécies (Figura 1A) e, em relação às épocas, houve uma distinção principalmente aos 15 dias após o acamamento das plantas (Figura 1B).

Os espectros também apresentaram ruídos instrumentais e desvios de linha de base, que provocaram o deslocamento do espectro na vertical e espalhamentos multiplicativos. Portanto, foi necessária a aplicação de métodos de pré-processamento e suavização para estes perfis espectrais. Segundo Souza ${ }^{28}$ variações sistemáticas nos espectros de reflectância podem mascarar o parâmetro de interesse e, assim, uma técnica de pré-processamento para os espectros deve ser empregada antes da construção dos modelos quimiométricos.

Foi utilizado para o conjunto de espectros o alisamento ou suavização por segunda derivada para a construção do modelo de fenólicos (Figura 2A), e $1^{\text {a }}$ derivada (Savitzky-Golay) e normalização vetorial (SNV) para flavonoides totais (Figura 2B). A remoção desses ruídos instrumentais aleatórios aumentou a razão sinal/ruído, auxiliando na análise dos dados e na construção das curvas de calibração. ${ }^{29}$ A partir dos dados obtidos via NIR e da aplicação de modelos matemáticos e estatísticos, foi possível encontrar nos espectros as regiões que, em princípio, se encontram os maiores teores de fenólicos e flavonoides. Os maiores picos de absorbância foram detectados nas regiões entre 3500 e $5500 \mathrm{~cm}^{-1}$ (Figura 2A) e entre 7500 e $9000 \mathrm{~cm}^{-1}$ (Figura 2B).

Após a etapa de pré-processamento espectral da matriz de dados, foram desenvolvidos, juntamente com os valores fornecidos pelos métodos de referências, os modelos de calibração multivariada. Foi utilizado o modelo de regressão por mínimos quadrados parciais, no qual foi estabelecida uma relação matemática quantitativa entre os dados obtidos pelo método de referência e os dados obtidos pelo NIR.
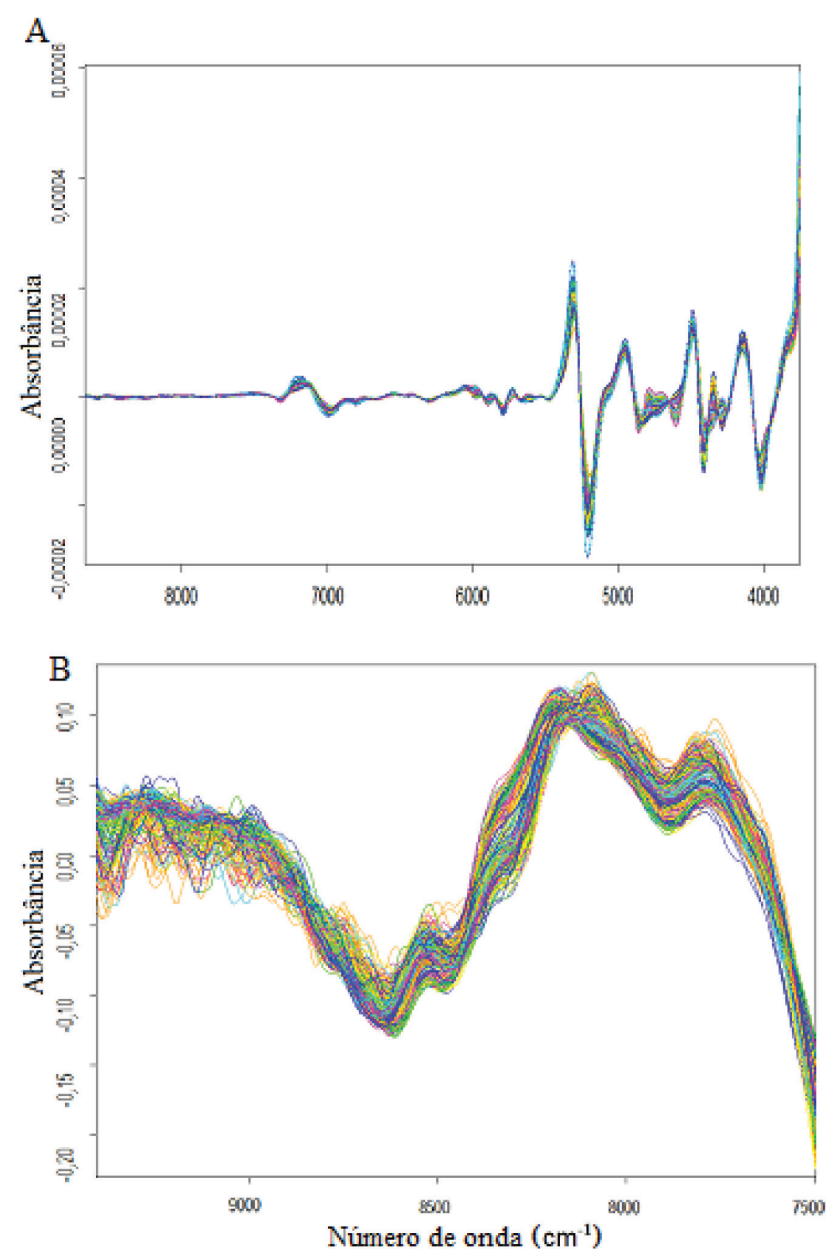

Figura 2. Conjunto dos espectros de NIR da parte aérea das espécies Raphanus sativus L., Secale cereale L. e Avena strigosa L. após o pré-processamento ( segunda derivada) para a construção das curvas de calibração de fenólicos totais (A) e ( $1^{a}$ derivada Savitzky-Golay e normalização vetorial (janela de 17 pontos) e de flavonoides totais (B)

Para a construção de modelos de regressão de fenólicos totais, foram utilizados os valores médios dos teores obtidos pelo método de Folin-Ciocalteau. Dos 252 espectros, correspondentes a 84 amostras das espécies de plantas de cobertura centeio, nabo-forrageiro e aveia-preta, foram utilizados 231 espectros na etapa de calibração (Tabela 1). Para flavonoides foram utilizados os valores médios dos teores obtidos pela metodologia descrita por Popova et al. ${ }^{23} \mathrm{e}$, dos 252 espectros, foram utilizados 234 espectros para a construção da curva de calibração (Tabela 1).

O conjunto de calibração, para os parâmetros químicos fenólicos e flavonoides totais, aplicando o método PLS, foi otimizado em função 
Tabela 1. Parâmetros de ajuste e erro dos modelos escolhidos para o conjunto de calibração para quantificação de compostos fenólicos e flavonoides totais

\begin{tabular}{lccccccc}
\hline & \multicolumn{5}{c}{ Conjunto de calibração } \\
\cline { 2 - 8 } Modelo & $\mathrm{N}^{\mathrm{o}}$ amostras & $\mathrm{N}^{\mathrm{o}}$ espectros & $\begin{array}{c}\mathrm{N}^{\mathrm{o}} \text { espectros } \\
\text { calibração }\end{array}$ & $\begin{array}{c}\text { Variáveis } \\
\text { latentes }\end{array}$ & $\begin{array}{c}\text { RMSECV } \\
\left(\mathrm{mg} \mathrm{g}^{-1}\right)\end{array}$ & $\mathrm{r}$ & Outliers \\
\hline Fenólicos & 84 & 252 & 231 & 4 & 1,53 & 0,93 & 7 \\
Flavonoides & 84 & 252 & 234 & 2 & 1,06 & 0,84 & 6 \\
\hline
\end{tabular}

do número de variáveis latentes, do coeficiente de correlação (r) e do erro médio quadrático do conjunto de calibração (RMSECV). O melhor modelo PLS obtido para o parâmetro fenólicos totais foi obtido utilizando 4 variáveis latentes com o coeficiente de correlação de 0,93 e 1,53 de RMSECV (Tabela 1). Frizon et al.,${ }^{4}$ ao determinarem o conteúdo de compostos fenólicos em 85 amostras de plantas de erva mate (Ilex paraguariensis) via NIR, obtiveram, nos diferentes modelos propostos, coeficientes de correlação próximos ao obtido neste estudo, entre 0,80 a 0,90 , e erros em torno de 20 a $22 \mathrm{mg} \mathrm{g}^{-1}$, dez vezes maior ao erro encontrado neste trabalho.

Em relação ao número de outliers, foram excluídas em média de 7 a $8 \%$ do total das amostras avaliadas (Tabela 1). A maioria dos trabalhos que utilizam o NIR para a construção de curvas tem excluído em torno de 2 a $8 \%$ do total de amostras. ${ }^{3,30,31}$ Foram eliminadas, portanto, as amostras que sofreram algum tipo de contaminação na obtenção dos espectros ou que se encontraram fora do intervalo previsto para os dados em questão. ${ }^{30}$

$\mathrm{Na}$ calibração, a relação entre as concentrações obtidas pelo método de referência versus as concentrações preditas de fenólicos encontraram-se na faixa de calibração de 0,4 a 21,0 $\mathrm{mg} \mathrm{g}^{-1}$ (Figura $3 \mathrm{~A})$. As amostras analisadas apresentaram concentrações mais frequentes no limite inferior, em torno de 0,4 a $9,0 \mathrm{mg} \mathrm{g}^{-1}$, que são principalmente as espécies analisadas aos 15 e 30 dias após o acamamento e as gramíneas aveia-preta e centeio. No limite superior, de 15 a $21 \mathrm{mg} \mathrm{g}^{-1}$, encontra-se predominantemente a espécie nabo-forrageiro (Figura 3A). As maiores concentrações encontradas no nabo-forrageiro podem ser atribuídas às características da espécie, que é conhecida por sua atividade alelopática e pela sua produção de compostos fenólicos..$^{32,33}$

Para o parâmetro flavonoides foram utilizadas 2 variáveis latentes com o coeficiente de correlação de 0,84 e 1,06 de RMSECV (Tabela 1). Trabalhos realizados por Sousa ${ }^{28}$ e Yong et al. ${ }^{34}$ ao determinarem o conteúdo de flavonoides via espectroscopia NIR combinada ao método de calibração multivariada em algodão e em folhas de Ginkgo biloba, também utilizaram o método PLS para a análise exploratória e construção de modelos, e obtiveram, respectivamente, concentrações de 80,3 a $383,5 \mu \mathrm{g} / 10 \mathrm{~mL}$ e 2,73 a $36,68 \mathrm{mg} \mathrm{g}^{-1}$, e erros em torno de $2,30 \mathrm{mg} \mathrm{g}^{-1} \mathrm{e} 32,7 \mathrm{mg} / 10 \mathrm{~mL}$, com coeficientes de correlação de 0,82 e 0,90. Entretanto, observa-se que os erros foram mais altos quando comparados com os resultados obtidos neste estudo. Segundo Morgano et al., ${ }^{35}$ para minimizar os erros de previsão e obter um RMSECV baixo, ou seja, diminuir a diferença entre os dados obtidos pelo método de referência e no NIR, é preciso escolher a região do melhor conjunto de números de onda do perfil espectral.

As concentrações obtidas pelo método de referência versus as concentrações preditas para flavonoides foram encontradas na faixa de calibração de 0,1 a 9,5 mg g-1. Na calibração, as amostras analisadas apresentaram concentrações mais frequentes no limite inferior, em torno de 0,1 a 3,5 $\mathrm{mg} \mathrm{g}^{-1}$ (Figura 3B) e, no limite superior, de 6,0 a $9,5 \mathrm{mg} \mathrm{g}^{-1}$, encontra-se predominantemente a espécie nabo-forrageiro (Figura 3B). Tanto para fenólicos como para flavonoides, os valores medidos e preditos das amostras com maior concentração do composto são oriundos da espécie nabo-forrageiro aos 60, 80 e 100 dias após o cultivo. Sousa, ${ }^{28}$ ao utilizar a espectroscopia NIR para construção de

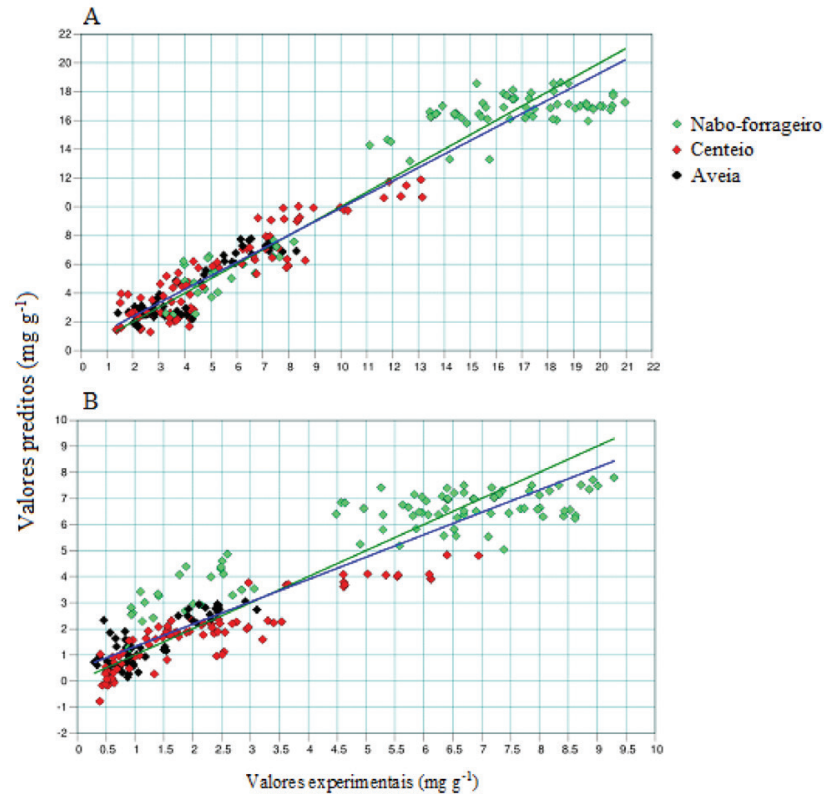

Figura 3. Curvas de calibração dos valores preditos pelo NIR versus os valores experimentais pelo método de referência de compostos fenólicos totais (A) e flavonoides $(B)\left(\mathrm{mg} \mathrm{g}^{-1}\right)$ aplicando o modelo de regressão por mínimos quadrados parciais (PLS)

curvas de calibração de flavonoides em amostras de algodão, observou que as amostras também se agrupavam em determinadas faixas de concentração da curva, neste caso de acordo com a pigmentação.

Após a construção dos modelos de calibração, também foi realizada a distribuição fatorial dos espectros obtidos via NIR com base na análise de PCA para detectar diferenças entre as espécies, épocas de avaliação e manejo das espécies (Figuras 4A, 4B e 4C).

Os eixos PC1 e PC2 explicam, em média, $83 \%$ e $16 \%$ da variabilidade dos dados, respectivamente. Observa-se, com a análise de PCA juntamente com a análise cluster hierárquico (Figura 5A), que ocorreu a separação da espécie nabo-forrageiro em relação às gramíneas aveia-preta e centeio, quando analisadas na região de 3757 a $8663 \mathrm{~cm}^{-1}$ do espectro. Nessa região encontram-se as maiores diferenças nos espectros dessas espécies, com combinações C-H e C-C que são típicas da estrutura química dos compostos fenólicos e flavonoides. ${ }^{36}$

Em relação às épocas avaliadas, houve uma separação das espécies aos 15 e aos 30 dias após o acamamento, diferindo das espécies avaliadas aos 60, 80 e 100 DAS (Figura 4B). Na análise de agrupamento hierárquico também é possível observar a separação das amostras avaliadas aos 15 e aos 30 DAA (Figura 5B).

A sobreposição das amostras entre as épocas de avaliação 60, 80 e 100 dias após a semeadura, em PC2(-) (Figura 4B), aponta uma similaridade entre essas amostras quando analisadas via NIR, sugerindo que os espectros das espécies centeio, nabo-forrageiro e aveia-preta, que se encontravam em estádio de desenvolvimento vegetativo e florescimento, podem ser mais semelhantes quando comparados com os 

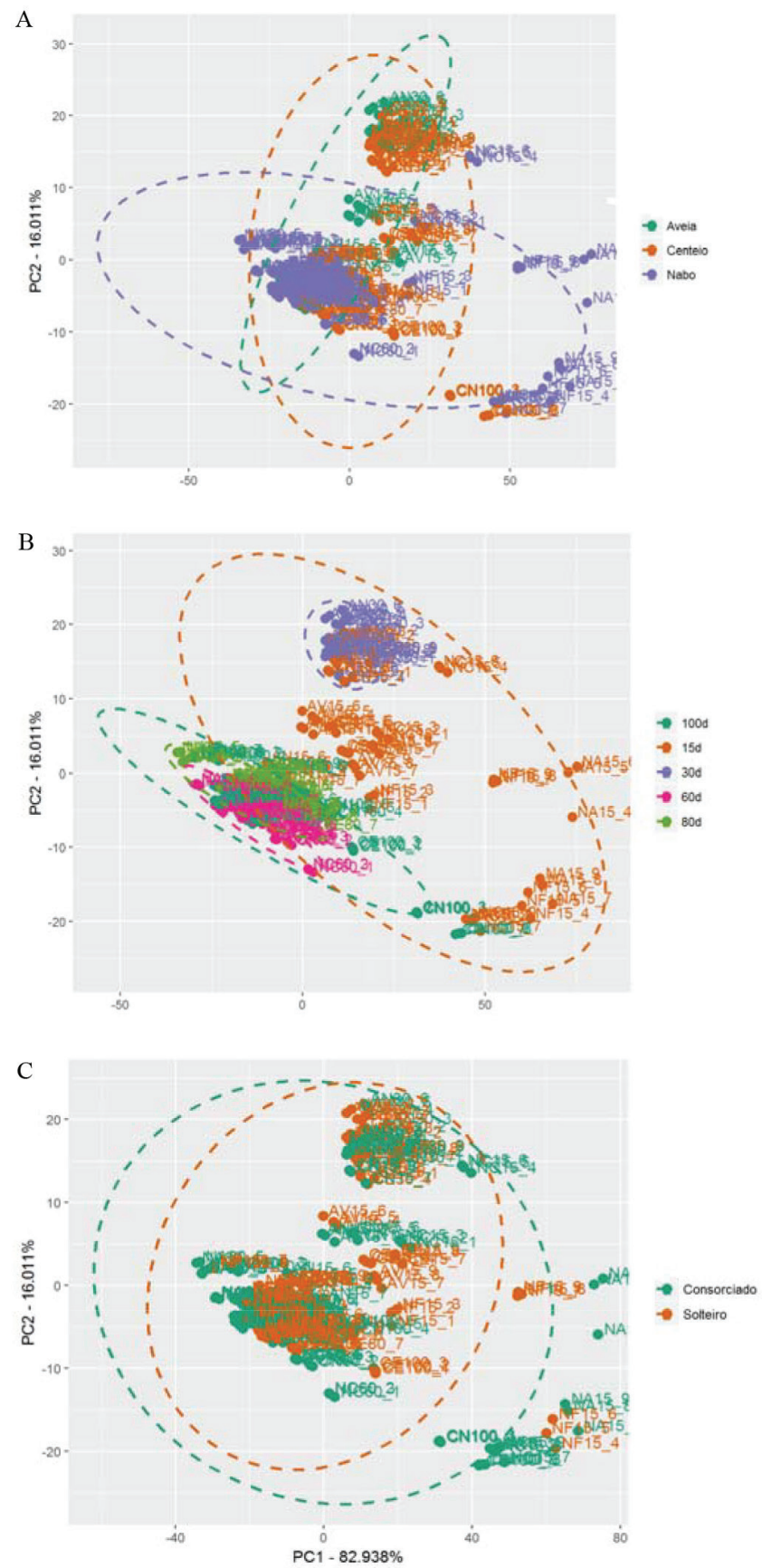

Figura 4. Distribuição fatorial dos componentes principais 1 (PC1) e 2 (PC2) dos espectros de reflectância na região do infravermelho (3500 a $\left.8700 \mathrm{~cm}^{-1}\right)$ das amostras sólidas, em relação às espécies de plantas de cobertura (A), em relação aos tempos de avaliação, aos 60, 80, 100 dias após a semeadura e 15 e 30 dias após o acamamento das plantas $(B)$ e em relação ao sistema de cultivo $(C)$

espectros obtidos após estas plantas serem acamadas no campo. Além disso, outros elementos estruturais da planta podem ter contribuído para essa separação nas épocas analisadas, como teores de celulose, a relação $\mathrm{C} / \mathrm{N}$, teores de lignina, entre outros elementos presente nas plantas. A espécie nabo-forrageiro, por apresentar uma relação $\mathrm{C} / \mathrm{N}$ menor e maior taxa de mineralização do material, é degradada nos 15 primeiros dias, ${ }^{37,38}$ e é provável que os compostos fenólicos já haviam sido liberados ou degradados. ${ }^{39}$

Quando as amostras foram analisadas em relação ao sistema de cultivo, não houve diferenças entre as espécies quando cultivadas
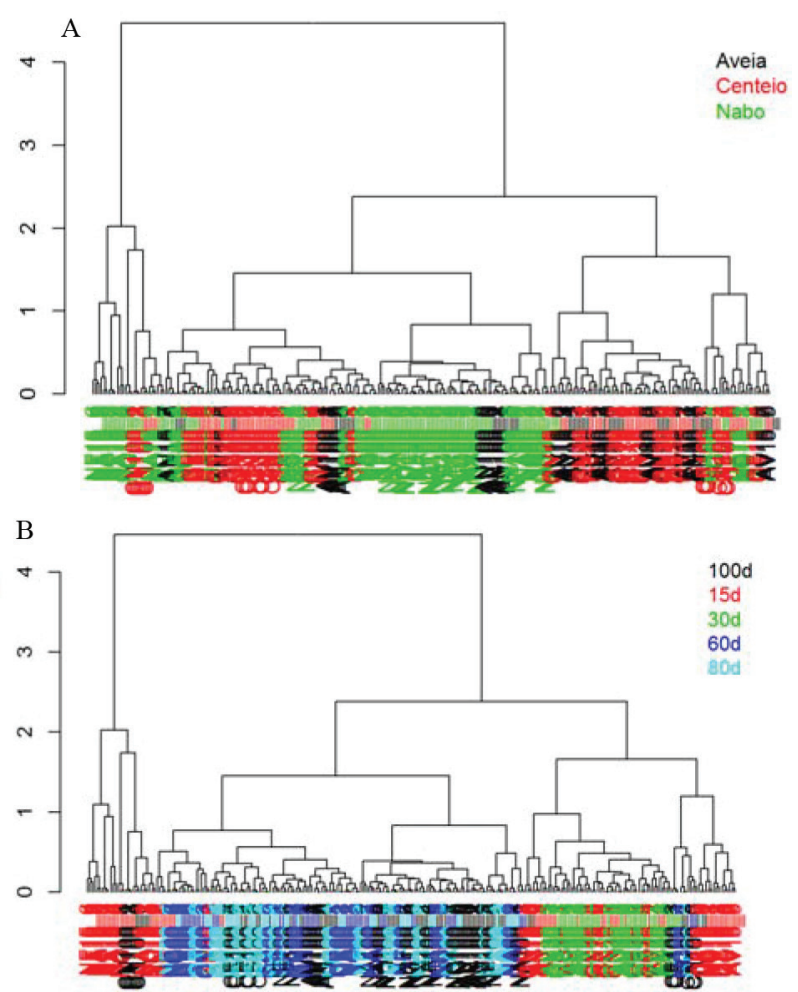

Figura 5. Dendograma dos espectros de reflectância obtido pela análise de cluster hierárquico utilizando distância euclidiana, em relação às espécies (A) e em relação às épocas de coleta $(B)$

solteiras e/ou consorciadas em relação aos espectros obtidos e a composição fenólica (Figura 4C).

\section{Validação do modelo}

Após a construção dos modelos de calibração para fenólicos e flavonoides totais, foi utilizado um conjunto de amostras não incluídas na calibração, mas semelhantes ao conjunto de calibração, para a escolha do melhor modelo para validação, conforme apresentado na Tabela 2. Foram utilizados um conjunto de 10 e 9 amostras e analisadas em triplicata para a quantificação de fenólicos e flavonoides, respectivamente.

Para fenólicos totais, o erro médio quadrático do conjunto de validação (RMSEP) foi de 1,44 e o coeficiente de correlação de 0,95 e, para flavonoides, o RMSEP foi de 0,77 e o coeficiente de correlação de 0,94 (Tabela 2). Yong et al. ${ }^{34}$ ao analisarem os teores de flavonoides em folhas de Ginkgo biloba, obtiveram um RMSEP de 2,62 $\mathrm{mg} \mathrm{g}^{-1}$, enquanto Frizon et $a l .{ }^{4}$, ao determinarem os teores de compostos fenólicos totais em folhas de erva mate, obtiveram o melhor modelo com um RMSEP de $12,12 \mathrm{mg} \mathrm{g}^{-1} \mathrm{e}$ um coeficiente de 0,81 .

Nas Tabelas 3 e 4 apresentam-se, para fins comparativos, os valores obtidos pelo método de referência, os valores obtidos pelo NIR, o resíduo e o erro percentual (\%) para os teores de fenólicos e flavonoides totais das amostras para a construção das curvas validação externa, respectivamente.

Na Figura 6A é possível observar a curva de calibração escolhida (linha azul) e de validação externa (linha verde) para fenólicos totais. Após a aplicação de métodos matemáticos e estatísticos, a curva de calibração foi escolhida considerando o menor erro obtido e de acordo com as regiões do NIR nas quais existiam as maiores diferenças entre os espectros e as amostras. Além disso, também foram observados os gráficos gerados pelo software, como a distância entre os espectros, rankings e o RMSECV. O erro relativo médio entre o método 
Tabela 2. Parâmetros de ajuste e erro dos modelos escolhidos para o conjunto de validação externa para quantificação de compostos fenólicos e flavonoides totais

\begin{tabular}{lccccc}
\hline \multirow{2}{*}{ Modelo } & \multicolumn{5}{c}{ Curva de validação externa } \\
\cline { 2 - 6 } & $\mathrm{N}^{\mathrm{o}}$ amostras & $\mathrm{N}^{\mathrm{o}}$ espectros & $\mathrm{N}^{\mathrm{o}}$ espectros validação & RMSEP $\left(\mathrm{mg} \mathrm{g}^{-1}\right)$ & $\mathrm{r}$ \\
\hline Fenólicos & 10 & 30 & 30 & 1,44 & 0,95 \\
Flavonoides & 9 & 27 & 27 & 0,77 & 0,94 \\
\hline
\end{tabular}

Tabela 3. Comparação dos resultados de compostos fenólicos totais $\left(\mathrm{mg} \mathrm{g}^{-1}\right)$ para as amostras do conjunto de validação externa obtido pelo método de referência e NIR aplicando o modelo de regressão por mínimos quadrados parciais de melhor ajuste obtido

\begin{tabular}{lcccc}
\hline & $\begin{array}{c}\text { Valor obtido } \\
\text { pelo método de } \\
\text { Amostra }\end{array}$ & $\begin{array}{c}\text { Valor previsto } \\
\text { pelo NIR }\end{array}$ & Resíduo & $\begin{array}{c}\text { Erro } \\
(\%)\end{array}$ \\
\cline { 2 - 3 } & $------------\mathrm{mg} \mathrm{g}^{-1}$------------ & & \\
\hline A+N 80d.1 & 8,9179 & 10,5310 & $-1,6131$ & 18,1 \\
A+N 80d.2 & 8,0012 & 10,5383 & $-2,5371$ & 31,7 \\
A+N 80d.3 & 8,4595 & 10,4524 & $-1,9929$ & 23,6 \\
Aveia 60 d.1 & 14,0714 & 15,4382 & $-1,3668$ & 9,7 \\
Aveia 60 d.2 & 15,619 & 15,4111 & 0,2079 & 1,3 \\
Aveia 60 d.3 & 12,2857 & 15,5568 & $-3,2711$ & 26,6 \\
Aveia 80 d.1 & 8,6560 & 10,4429 & $-1,7869$ & 20,6 \\
Aveia 80 d.2 & 8,1976 & 11,0707 & $-2,8731$ & 35,0 \\
Aveia 80 d.3 & 7,7393 & 10,8302 & $-3,0909$ & 39,9 \\
C+N 60d.1 & 12,3700 & 12,4109 & $-0,0409$ & 0,3 \\
C+N 60d.2 & 12,2300 & 12,3679 & $-0,1379$ & 1,1 \\
C+N 60d.3 & 10,5900 & 12,2141 & $-1,6241$ & 15,3 \\
Centeio 60d.1 & 11,0000 & 10,1699 & 0,8301 & $-7,5$ \\
Centeio 60d.2 & 11,2700 & 10,3378 & 0,9322 & $-8,3$ \\
Centeio 60d.3 & 9,9000 & 10,4397 & $-0,5397$ & 5,5 \\
Centeio 100.1 & 4,2600 & 4,4997 & $-0,2397$ & 5,6 \\
Centeio 100.2 & 4,7200 & 4,8970 & $-0,177$ & 3,8 \\
Centeio 100.3 & 5,3200 & 5,0965 & 0,2235 & $-4,2$ \\
N+A 80d.1 & 17,7100 & 16,8399 & 0,8701 & $-4,9$ \\
N+A 80d.2. & 16,6200 & 16,8047 & $-0,1847$ & 1,1 \\
N+A 80d.3 & 18,8100 & 16,7693 & 2,0407 & $-10,8$ \\
N+A 100d.1 & 13,7600 & 13,7474 & 0,0126 & $-0,1$ \\
N+A 100d.2 & 14,0600 & 13,7693 & 0,2907 & $-2,1$ \\
N+A 100d.3 & 13,9100 & 13,7922 & 0,1178 & $-0,8$ \\
Nabo 60d.1 & 15,7200 & 15,8615 & $-0,1415$ & 0,9 \\
Nabo 60d.2 & 17,8300 & 15,8432 & 1,9868 & $-11,1$ \\
Nabo 60d.3 & 14,3600 & 15,7185 & $-1,3585$ & 9,5 \\
Nabo 80d.1 & 18,6700 & 18,8015 & $-0,1315$ & 0,7 \\
Nabo 80d.2 & 18,8100 & 18,7529 & 0,0571 & $-0,3$ \\
Nabo 80d.3 & 18,5300 & 18,6351 & $-0,1051$ & 0,6 \\
\hline
\end{tabular}

quimiométrico e o método de referência foi de $\pm 10 \%$ (Tabela 3 ), o que indica uma boa previsão dos teores fenólicos totais pelo método proposto comparando-se com outros métodos. ${ }^{3,4,28}$ Entretanto, algumas amostras apresentaram diferenças um pouco maiores entre os valores obtidos pelo método de referência e o método proposto, como a espécie aveia aos 80 dias e quando consorciada com o nabo aos 80 dias de avaliação (A+N 80d), que apresentaram erros de 18 até $39 \%$ (Tabela 3). Estas amostras não foram retiradas da validação externa, pois os valores de RMSEP e o coeficiente de correlação pouco se alteraram quando retiradas da curva. Essas diferenças observadas entre o método de referência e o método NIR podem ser atribuídas ao método de extração e ao uso de solventes para a extração de fenólicos, pois as análises realizadas no UV-vis, pelo método de Folin-Ciocalteau, já
Tabela 4. Comparação dos resultados de flavonoides totais $\left(\mathrm{mg} \mathrm{g}^{-1}\right)$ para as amostras do conjunto de validação externa obtido pelo método de referência e NIR aplicando o modelo de regressão por mínimos quadrados parciais de melhor ajuste obtido

\begin{tabular}{|c|c|c|c|c|}
\hline \multirow[t]{2}{*}{ Amostra } & $\begin{array}{l}\text { Valor obtido } \\
\text { pelo método de } \\
\text { referência }\end{array}$ & $\begin{array}{l}\text { Valor previsto } \\
\text { pelo NIR }\end{array}$ & \multirow[t]{2}{*}{ Resíduo } & \multirow[t]{2}{*}{$\begin{array}{l}\text { Erro } \\
(\%)\end{array}$} \\
\hline & \multicolumn{2}{|c|}{ 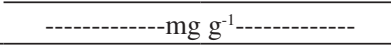 } & & \\
\hline Aveia 60d.1 & 5,2800 & 6,6739 & $-1,3939$ & 26,4 \\
\hline Aveia 60d.2 & 5,0943 & 6,7272 & $-1,6329$ & 32,1 \\
\hline Aveia 60d.3 & 5,4717 & 6,6739 & $-1,2022$ & 22,0 \\
\hline $\mathrm{C}+\mathrm{N}$ 60d.1 & 5,1200 & 5,3099 & $-0,1899$ & 3,7 \\
\hline $\mathrm{C}+\mathrm{N} 60 \mathrm{~d} .2$ & 5,3700 & 5,2045 & 0,1655 & $-3,1$ \\
\hline $\mathrm{C}+\mathrm{N} 60 \mathrm{~d} .3$ & 4,4500 & 5,1625 & $-0,7125$ & 16,0 \\
\hline Centeio 60d.1 & 4,6100 & 4,6443 & $-0,0343$ & 0,7 \\
\hline Centeio 60d.2 & 4,9500 & 4,4264 & 0,5236 & $-10,6$ \\
\hline Centeio 60d.3 & 4,6100 & 4,5403 & 0,0697 & $-1,5$ \\
\hline Centeio 100.1 & 1,2500 & 1,4670 & $-0,217$ & 17,4 \\
\hline Centeio 100.2 & 1,7600 & 1,7170 & 0,043 & $-2,4$ \\
\hline Centeio 100.3 & 1,9200 & 1,8897 & 0,0303 & $-1,6$ \\
\hline $\mathrm{N}+\mathrm{A}$ 80d.1 & 8,2300 & 8,5042 & $-0,2742$ & 3,3 \\
\hline $\mathrm{N}+\mathrm{A} 80 \mathrm{~d} .2$ & 8,9800 & 8,6010 & 0,379 & $-4,2$ \\
\hline $\mathrm{N}+\mathrm{A} 80 \mathrm{~d} .3$ & 8,3900 & 8,5638 & $-0,1738$ & 2,1 \\
\hline $\mathrm{N}+\mathrm{A}$ 100d.1 & 4,5500 & 3,8157 & 0,7343 & 16,1 \\
\hline $\mathrm{N}+\mathrm{A} 100 \mathrm{~d} .2$ & 4,0300 & 3,7781 & 0,2519 & 6,3 \\
\hline $\mathrm{N}+\mathrm{A} 100 \mathrm{~d} .3$ & 4,0400 & 3,7851 & 0,2549 & 6,3 \\
\hline Nabo 60d.1 & 6,9700 & 6,8587 & 0,1113 & $-1,6$ \\
\hline Nabo 60d.2 & 8,2300 & 7,0015 & 1,2285 & $-14,9$ \\
\hline Nabo 60d.3 & 7,6000 & 6,9974 & 0,6026 & $-7,9$ \\
\hline Nabo 80d.1 & 9,190 & 7,9375 & 1,2525 & $-13,6$ \\
\hline Nabo 80d.2 & 8,710 & 8,0136 & 0,6964 & $-8,0$ \\
\hline Nabo 80d.3 & 8,230 & 7,9595 & 0,2705 & $-3,3$ \\
\hline $\mathrm{N}+\mathrm{C} 80 \mathrm{~d} .1$ & 6,710 & 4,9756 & 1,7344 & 25,8 \\
\hline $\mathrm{N}+\mathrm{C} 80 \mathrm{~d} .2$ & 6,210 & 5,2831 & 0,9269 & 14,9 \\
\hline $\mathrm{N}+\mathrm{C} 80 \mathrm{~d} .3$ & 5,030 & 5,3499 & $-0,3199$ & 6,4 \\
\hline
\end{tabular}

se tratavam de um produto da extração dos compostos com metanol, podendo extrair e evidenciar mais os erros obtidos pelo método e os teores de fenólicos quando comparados com o NIR.

Para flavonoides, os erros relativos médios entre o método quimiométrico e o método de referência também foram de $10 \%$; exceção foi a amostra aveia 60d, que apresentou erros maiores, de 22 a $32 \%$ (Tabela 4). Como se tratam de valores baixos, de 1,5 a 9,0 $\mathrm{mg} \mathrm{g}^{-1}$, quaisquer erros obtidos nas menores concentrações serão mais perceptíveis do que quando comparados com valores mais altos, como 18 e $20 \mathrm{mg} \mathrm{g}^{-1}$. Frizon et al., ${ }^{4}$ ao determinarem o conteúdo de compostos fenólicos em erva mate via NIR e método de referência, também obtiveram maiores discrepâncias em relação às amostras com menores concentrações.

Na Figura 6B é possível observar a correlação entre os valores médios do teor de flavonoides do conjunto de amostras obtidos pelo NIR e os valores experimentais dos 27 espectros utilizados no 


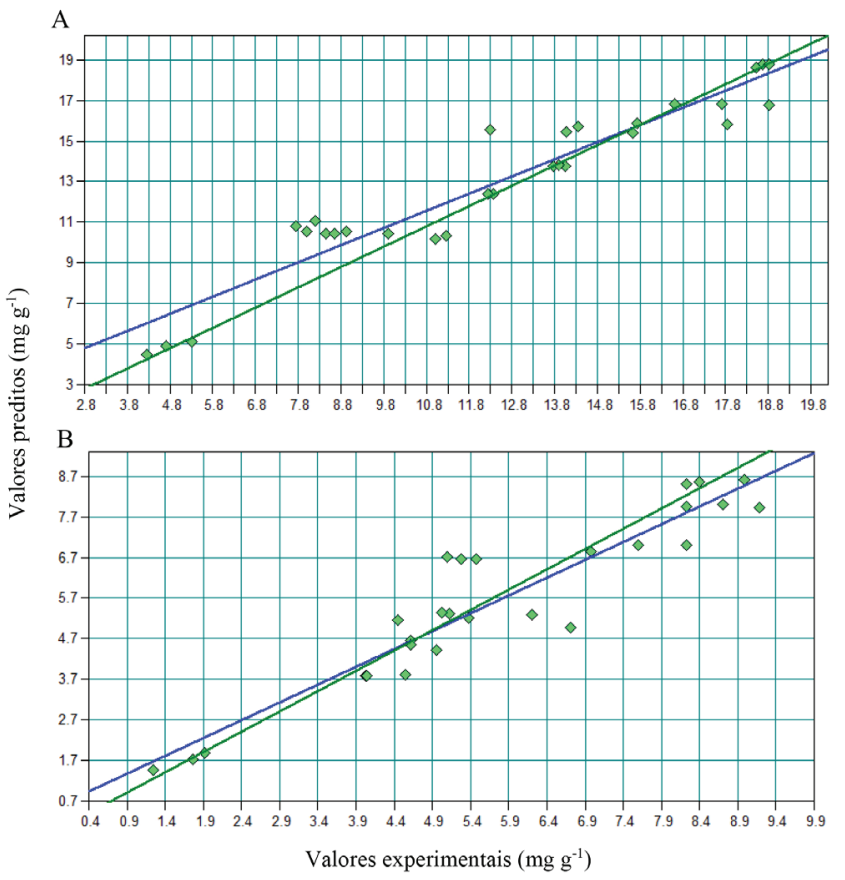

Figura 6. Correlação entre os valores médios previstos pelo NIR versus valores médios obtidos pelo método de referência para o conjunto de validação externa do modelo para compostos fenólicos totais (A) e flavonoides (B)

conjunto de validação externa. Lima et al., ${ }^{12}$ ao avaliarem os teores de nitrogênio total em amostras de folhas de trigo (Triticum spp.) para um modelo de validação externa, obtiveram um erro médio de \pm $3 \%$. Nos trabalhos realizados por Morgano et al., ${ }^{35}$ ao determinarem o teor de proteína total em amostras de café (Coffea sp) cru via NIR e pelo método PLS, os autores obtiveram o melhor modelo PLS com um erro médio de $7 \%$ entre o método quimiométrico e o método de referência. Mazur et $a l .{ }^{3}$ também obtiveram um erro médio de $7 \%$ entre o método NIR e o de referência para a quantificação de metilxantina em erva mate.

Dentre as amostras utilizadas no teste de validação, dois terços apresentaram um erro de previsão inferior a 10\%, e a baixa dispersão dos valores de previsão em torno das curvas, utilizando apenas 9 e 10 amostras para a validação externa, auxiliou na construção dos modelos de previsão. Contudo, as maiores discrepâncias ocorreram em relação à amostra aveia.

\section{CONCLUSÃO}

Recomenda-se a espectroscopia NIR para a análise exploratória e discriminatória de amostras e para o desenvolvimento dos modelos de calibração por permitir inferir, de forma não destrutiva, os teores de fenólicos e flavonoides totais, com alta frequência analítica, sem o uso de reagentes de alto custo e doutros insumos requeridos, com resultados muito próximos aos obtidos pelo método de referência.

A combinação da espectroscopia NIR e análise multivariada por regressão por mínimos quadrados parciais permitiu o desenvolvimento de modelos de previsão de fenólicos e flavonoides com erros associados em média de $10 \%$.

A análise exploratória dos espectros, por meio da aplicação de métodos quimiométricos, discriminou a espécie nabo-forrageiro das espécies de plantas de cobertura aveia-preta e centeio em relação ao perfil químico.

Entre os tempos de avaliação houve uma discriminação das amostras, sendo mais evidente aos 15 dias de avaliação, quando todas as espécies já haviam sido acamadas no campo, em relação aos tempos em que as plantas se encontravam em estádio de desenvolvimento vegetativo e florescimento.

\section{REFERÊNCIAS}

1. Silva, M. L. C.; Costa, R. S.; Santana, A. S.; Koblitz, M. G. B.; Semina: Cienc. Agrar. 2010, 31, 669.

2. Liu, Y.; Gamble, G.; Thibodeaux, D.; Trans. ASABE 2010, 53, 1341.

3. Mazur, L.; Peralta-Zamora, P. G.; Demczuk, B.; Ribani, R. F.; J. Food Compos. Anal. 2014, 35, 55.

4. Frizon, C. N. T.; Oliveira, G. A.; Perussello, C. A.; Peralta-Zamora, P. G.; Camlofski, M. O.; Rossa, U. B.; Hoffmann-Ribani, R.; LWT--Food Sci. Technol. 2015, 60, 795.

5. Sánchez, M. T.; Pérez-Martins, D.; Torres, I.; Gil, B.; Garrido-Varo, A.; Haba, M. J.; Postharvest Biol. Technol. 2017, 125, 122.

6. Pasquini, C. J.; Braz. Chem. Soc. 2003, 14, 198.

7. Rohman, A.; Man, Y. B. C.; Food Chem. 2011, 129, 583.

8. Ferreira, D. S.; Poppi, R. J.; Pallone, J. A. L.; J. Cereal Sci. 2015, 64, 43.

9. Kim, J. K.; Kim, E. H.; Lee, O. K.; Park, S. Y.; Lee, B.; Kim, S. H.; Park, I.; Chung, I. M.; Food Chem. 2013, 141, 2988.

10. Maraschin, M.; Zeggio, A. S.; Oliveira, S. K.; Kunhnen, S.; Tomazzoli, M. M.; Raguzzoni, J. C.; Zeri, A. C. M.; Carreira, R.; Correia, S. Costa, C.; Rocha, M.; J. Nat. Prod. 2015, 79, 13.

11. Jandric, Z.; Cannavan, A.; Food Control. 2017, 72, 173.

12. Lima, K. M. G; Trevisan, M. G; Poppi, R. J; Andrade, J. C.; Quim. Nova 2008, 31, 700 .

13. Felix, J. C.; Vendrame, P. R. S.; Marchão, R. L.; Oliveira, J. F.; Guimarães, M. F.; Brossard, M.; Becquer, T.; Brito, O. R.; Pesq. Agropec. Bras. 2016, 51, 1405.

14. Luca, F.; Conforti, M.; Castrignano, A.; Matteucci, G.; Buttafuoco, G.; Geoderma 2017, 288, 175.

15. Neto, B. B., Scarmínio, I. S., Bruns, R. E.; Quim. Nova. 2006, 29, 1401.

16. Sabir, A.; Rafi, M.; Darusman, L. K.; Food Chem. 2017, 221, 1717.

17. Pires, F. F.; Prates, E. R.; R. Bras. Zootec. 1998, 33, 1076.

18. Taiz, L.; Zeiger, E.; Fisiologia vegetal, $4^{\mathrm{a}}$ ed., ARTMED: Porto Alegre, 2009.

19. Finney, M. M.; Danehower, D. A.; Burton, J. D.; J. Chromatogr. A. 2005 , 1066, 249.

20. Labbafy, M. R.; Maighny, F.; Hejazy, A.; Khalaj, H.; Baghestany, A. M.; Allahdady, I.; Mehrafarin, A.; Am.-Eurasian J. Agric. Environ. Sci. 2009, 1,25 .

21. Ediage, E. N.; Mavungu, J. D. D.; Scippo, M. L.; Schneider, Y. J.; Larondelle, Y.; Callebaut, A.; Robbens, J.; Peteghem, C. V.; Saeger, S. D.; J. Chromatogr. A. 2011, 1218, 4395.

22. Singleton, V. L.; Rossi, J. A.; Amer. J. Enol. Viticult. 1965, 16, 144.

23. Popova, M.; Bankova, V. S,; Butovska, D.; Petkov, V.; NikolovaDamyanova, B.; Sabatini, A. G.; Marcazzan, G. L.; Bogdanov, S.; Phytochem. Anal. 2004, 15, 235.

24. Opus Lab Bruker ${ }^{\circledR}$ software (v. 7.5), 2014.

25. R Development Core Team. R: A Language and Environment for Statistical Computing. R Foundation for Statistical Computing, Vienna, Austria, 2010.

26. Costa, C; Maraschin, M; Rocha, M.; specmine: Metabolomics and Spectral Data Analysis and Mining. R package version 1.0. 2015.

27. Souza, A. M.; Breitkreitz, M. C.; Filgueiras, P. R.; Rohwedder, J. J. R.; Poppi, R. J.; Quim. Nova. 2013, 36, 1057.

28. Sousa, G. R. M.; Dissertação Mestrado, Universidade Estadual da Paraíba, Brasil, 2013.

29. Neves, A. C. O.; Soares, G. M.; De Morais, S. C.; De Costa, F. S. L., Porto, D. L.; De Lima, K. M. G. J.; Pharm. Biomed. Anal. 2012, 57, 115.

30. Viana, L. C.; Dissertação Mestrado, Universidade Federal de Lavras, Brasil, 2008.

31. Santos, R. B.; Gomide, J. L.; Souza, L. C.; Rev. Árvore. 2009, 33, 259. 
32. Cartea, M. E.; Francisco, M.; Soengas, P.; Velasco, P.; Molecules 2011 16, 251.

33. Rehman, M. U.; Hussain, M.; Ali, M.; Mustafa, C. B.; Shafi, J.; Iqbal, F.; Sci. Agric. 2013, 3, 46.

34. Yong, S. J.; Bo, Z. X.; Wen, Z. J.; Holmes, M.; Liang, W. K.; Xue, W.; Hong, C.; Acta Part. 2012, 94, 271.

35. Morgano, M. A.; Faria, C. G.; Ferrão, M. F.; Bragagnolo, N.; Ferreira, M. M. C.; Ciênc. Tecnol. Aliment. 2005, 25, 21.

36. Burns, J.; Gardner, P. T.; Matthews, D.; Duthie, G. G.; Lean, M. E.; Crozier, A.; J. Agric. Food Chem. 2001, 49, 5797.
37. Martins, R. P.; Comin, J. J.; Gatiboni, L. C.; Soares, C. R. F.; Couto, R. R.; Brunetto, G. Rev. Ceres. 2014, 61, 587.

38. Oliveira, R. A.; Brunetto, G.; Loss, A.; Gatiboni, L. C.; Kurtz, C.; Muller, V. M.; Lovato, P. E.; Oliveira, B. S.; Souza, M.; Comin, J. J.; Rev. Bras. Ciênc. Solo 2016, 40, 1.

39. Moreira, F. M. S.; Siqueira, J. O.; Microbiologia e bioquímica do solo, $2^{a}$ ed., Ed. UFLA: Lavras, 2006. 